\title{
Correction to: Cybersecurity Threats, Vulnerability and Analysis in Safety Critical Industrial Control System (ICS)
}

\author{
Xinxin Lou and Asmaa Tellabi
}

\section{Correction to:}

\section{Chapter "Cybersecurity Threats, Vulnerability} and Analysis in Safety Critical Industrial Control System (ICS)" in: E. Pricop et al. (eds.), Recent Developments on Industrial Control Systems Resilience, Studies in Systems, Decision and Control 255, https://doi.org/10.1007/978-3-030-31328-9_4

The original version of the book was inadvertently published with an incorrect figure 2 in Chapter "Cybersecurity Threats, Vulnerability and Analysis in Safety Critical Industrial Control System (ICS)". The figure 2 is updated with the correct figure given below and statement has been updated at page number 78 as "As for the safety critical system, we tend to consider the integrity as a first". The chapter and book have been updated with the changes.

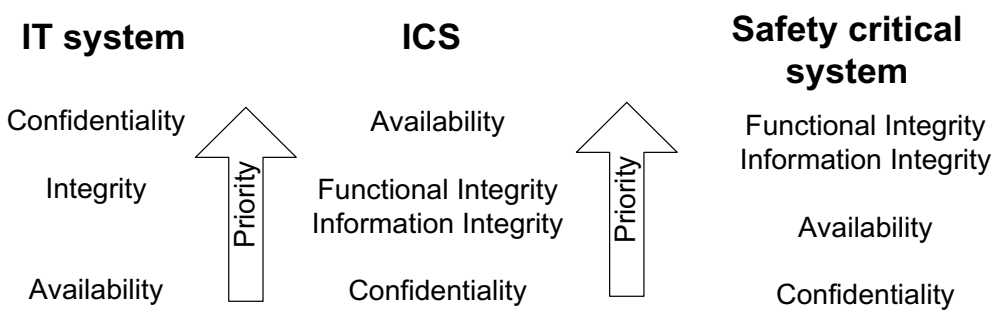

Fig. 2 Comparison of security objectives priority among various systems

\footnotetext{
The updated version of this chapter can be found at https://doi.org/10.1007/978-3-030-31328-9_4 